\title{
Low-dose Polyethylenimine-Mediated Transfection to Generate Human Induced Pluripotent Stem Cell: an optimized protocol
}

\section{Monir Shayestehfar}

Iran University of Medical Sciences

\section{Sara Farahi}

Shahid Beheshti University of Medical Sciences

\section{Behjat Kheiri Yeganeh Azar}

Iran University of Medical Sciences

\section{Amirhossein Memari}

Tehran University of Medical Sciences

Tourandokht Baluchnejadmojarad

Iran University of Medical Sciences

Faeze Faghihi ( $\nabla$ faezefaghihi@yahoo.com )

Iran University of Medical Sciences

\section{Research Article}

Keywords:

Posted Date: February 15th, 2022

DOI: https://doi.org/10.21203/rs.3.rs-1314449/v1

License: (9) This work is licensed under a Creative Commons Attribution 4.0 International License. Read Full License 


\section{Abstract}

Human dermal fibroblasts (HDF) can be reprogrammed through different strategies to generate human induced pluripotent stem cells (hiPSCs). However, most of these strategies require high-cost materials and specific equipment not readily accessible in most laboratories. Hence, liposomal and virus-based techniques can replace with polyethyleneimine (PEI)-mediated transfection to overcome these challenges. However, few researchers have addressed the PEl's ability to transfect HDFs. This study used PEI reagent to transfer oriP/EBNA1-based vector into HDFs to produce hiPSC lines. We first described conditions allowing the efficient transfection of HDFs with low cytotoxicity and without specific types of equipment and optimized several parameters relevant to the transfection procedure. We then monitored the effect of different N/P ratios on transfection efficiency and cytotoxicity using flow cytometry and fluorescent microscopy. By the results, we found that transfection efficiency was greatly affected by plasmid DNA (pDNA) concentration, PEI concentration, order of combining reagents, serum presence in polyplexes, and the duration of serum starvations. Moreover, using the optimized condition, we found that the N/P ratio of 3 achieved the highest percentage of HDFs positive for pGFP $(\sim 40 \%)$ with minimal cell toxicity. We finally generated hiPSCs using the optimized protocol and oriP/EBNA1-based vectors. We confirmed hiPSC formation by characterizing tests: Alkaline Phosphatase (AP) staining, immunocytochemistry (ICC) assay, real-time PCR analysis, in vitro differentiation into three germ layers, and karyotyping test. In conclusion, our results indicated that $25 \mathrm{kD}$ branched PEI could efficiently transfect HDFs toward generating hiPSCs via a simple, cost-effective, and optimized condition.

\section{Introduction}

Induced pluripotent stem cells (iPSCs) have opened great perspectives for disease modeling, high throughput drug discovery, and cell therapy in a patient-specific manner[1]. The genetic reprogramming of somatic cells toward a stem-cell-like state overcomes both the limitations of adult stem cells and the ethical issues encircling ESC use. In this approach, reprogramming of somatic cells occurs through forced and ectopic expression of defined pluripotency transcription factors named OCT4, SOX2, NANOG, KLF4, and $\mathrm{CMYC}$ [2]. Although iPSCs provide an assurance platform for generating disease models and drug screening, the clinical application of the iPSCs has been limited due to the lack of safe and efficient pluripotency gene delivery systems [3]. Several strategies can be used to induce pluripotency in somatic cells, including utilizing viral vectors, non-viral vectors, small molecules, RNAs, and proteins. Each method of generating iPSCs has its advantages and disadvantages. For instance, viruses are well-known delivery carriers with high efficient gene transfer and long-term expression potential. However, integrative properties of viral vectors arise concerns about mutations and safety[4]. Hence, using viral vectors is not recommended in clinical therapies [5]. Several scientists attempted to develop novel strategies to establish non-integrative methods. These methods are classified into two categories; non-integrating viral vectors like Adeno and Sendai viruses-based vectors and non-viral vectors such as various nano-carrier systems. In the last two decades, there has been a growing interest in nanoparticle delivery systems for generating iPSCs [6] due to their great potential to enhance stem cell and biology research[7, 8]. Among these vectors, cationic lipids are commonly administered in the iPSC technique. However, the cost of such commercial 
reagents such as Lipofectamine can be a limiting factor for labs facing financial problems. In addition, several other successful nanoparticulated delivery vehicles have been introduced in the literature for generating iPSCs including polyketal nanoparticles, cationic bolaamphiphile, Poly-b-amino esters, and Calcium phosphate nanocomposite[9-11] (See [8] for a comprehensive review) Although the research on a greater understanding of such nanoparticles has being conducted with promising results, the optimized protocol and the properties enabling these nanoparticles to overcome several barriers to intracellular DNA delivery still need further investigations[12, 13]. For example, in spite of the successful reprogramming ability of poly-b-amino esters, integrated transgenes were found in clones of hiPSCs and further investigations are still required to evaluate the feasibility of poly-b-amino ester-plasmid as a non-viral reagent[8]. Moreover, evidence have shown more potency of PEI for delivering genes of interest into cells in comparison to to one of the most common nanoparticulated delivery vehicles; calcium phosphate [14].

Despite common usage of different non-viral reagents for generating iPSCs from a wide range of somatic cells, cationic polymers such as polyethylenimine (PEI) which is one of the golden standard reagents for nucleic acid delivery applications[15, 16], have been less investigated in iPSC technology. Cationic polymers are attracting considerable interest due to their easy generation, the capability of large scales transfection, the versatility of DNA-polymer conjugation, long storage stability, and economic cost. PEI belongs to one of the most studied and effective families of manufactured polycations, which are available with different sizes (KDa) and structures (linear or branched) depending on the particular synthesis procedure [17]. The PEl's ability to form complexes with nucleic acids and carry them into the nucleus has been widely documented in vivo and in vitro conditions [18]. PEl and its derivatives have a high concentration of positively charged nitrogen atoms giving them a considerable buffering capacity due to better condensation of large negatively charged molecules such as DNA [19]. The significant buffering capacity of PEI led to the proton sponge hypothesis which describes that unprotonated amines of PEI can absorb protons facilitating the subsequent release of polyplexes into the cytoplasm[20]. In addition, Wang et al. showed that the total amount of pDNA within the cytosol was similar among cultured cells after transfection, showing PEl's prominent ability to penetrate cell membranes[21]. Seo et al. also evaluated the efficiency of PLAG/PEI/DNA nanoparticles in transfection and virus production in comparison with liposome-DNA complexes[22]. The authors showed superior transfection efficiency and viral yield in PLAG/PEI/DNA group when compared with liposome-DNA complexes, particularly in multiple gene transfection. These properties make PEI a good alternative for the delivery of desired genes and nucleic acids $[23,24]$.

However, despite the known considerable strength of PEI in the cell transfection process, not much attention has been given to the application of PEI in iPSC generation, which is a novel and gold strategy in personalized as well as regenerative medicine. In a comparable attempt, Drozd et al. investigated the generation of hiPSCs from cells of fibroblastic and epithelial origin by means of episomal reprogramming vectors via four reagents; Lipofectamine, PEI, FuGENE6, and FuGENE HD[25]. At the preliminary stages, they observed the highest expression levels achieved from PEI and FuGENE6 reagents[25]. Although PEI gave the highest transfection efficiency, the authors decided to use FuGENE6 for further experiments in their study due to lesser cytotoxic effects. 
Therefore, PEI dose-dependent toxicity on cells and tissues has remained unclear and controversial [26]. The cytotoxicity and efficiency of PEI have been linked to PEl's physicochemical properties such as molecular weight, branching ratio, and particle size[27, 28]. Most techniques have focused on refining PEI size, branch density, and deacylation to reduce PEI-mediated cell toxicity. But these methods are laborintensive and time-consuming $[29,30]$. Besides such methods, the way the polyplex is prepared can also significantly impact transfection efficiency and cytotoxicity [31-34]. So considering several parameters such as DNA amount, PEl amount, the ratios of moles of the amine groups of cationic polymers to those of the phosphate ones of DNA (N/P ratio) assists further optimizing transfection efficiency and cytotoxicity of polyplexes. To better clarify the properties of PEI/ plasmid DNA (pDNA) complexes for generating hiPSCs from Human Dermal Fibroblast (HDF), we intended to optimize several conditions regarding PEI/pDNA complexes. Herein, we investigated the influence of different parameters relevant to the formation of PEIpDNA complex, such as pDNA concentration, PEl concentration, order of combining reagents (i.e., PEl, pDNA), serum content of polyplexes, and serum starvation of cells before transfection. Then, after optimizing the polyplex preparation, we studied the influence of N/P ratio on HDF transfection to find the most efficient protocol for generating hiPSC lines in which 25KDa branched PEl and oriP/EBNA1-based vectors was used. Therefore, the main goal of the present study was to develop an efficient protocol for the PEI-mediated transfection of HDF toward hiPSCs comparable in ease to other non-viral commercial transfection reagents. We believe that our findings provide experimental evidence that could be usefully employed in other experiments in the area of safe and cost-effective iPSC generation.

\section{Results}

\section{2-1- Determining the best condition for transfection of HDFs}

Based on our previous experiments, PEI25KD at N/P ratio of 12 could efficiently transfect standard cell lines such as Human Embryonic Kidney (HEK) cells. Thus in the current study, different experiments conditions were adjusted based on PEI/pGFP ratio of 12 at the first stand. Fluorescent microscopy, flow cytometry, and MTT assay were performed to find the most efficient condition with few cytotoxicity effects. The following parameters were set and lead to find an optimized condition for PEl-mediated transfection (See Fig. 1 \& Table 1):

1. Plasmid concentration:

The fluorescence microscopy and flow cytometry method showed that the PEI/pDNA complex with $1 \mu \mathrm{g}$ pDNA efficiently transfected HDFs. In contrast, using $2 \mu \mathrm{g}$ pDNA decreased the transfection efficiency. Moreover, the HDF samples treated by PEI/pDNA complex containing $2 \mu$ DNA showed less than $70 \%$ cell viability.

2. PEl concentration:

We denoted that re-diluting PEl in distilled water before complex formation to reach $40 \mu \mathrm{g} / \mathrm{ml}$ concentration considerably increased transfection efficiency and cell viability compared to concentrated PEI $(1 \mathrm{mg} / \mathrm{ml})$. 
3. The sequence of adding reagents:

Our results showed that the order of adding reagents affects the efficiency of transfection. Adding PEI to pDNA increased the transfection efficiency while the vice versa order decreased the rate of transfection. However, the cell viability percentage did not differ significantly between samples treated by PEI/pDNA vs. pDNA/PEI complexes.

4. $10 \%$ FBS treatment during PEI/pDNA complexation:

MTT assay showed that FBS significantly reduced the cytotoxicity of PEI/pDNA complexes. However, fluorescent microscopy and flow cytometry assay determined that the presence of the FBS in polyplexes environment during complex formation dramatically decreased the transfection efficiency.

5. HDFs serum starvation before transfection:

Based on the fluorescent microscopy and flow cytometry results, 1 and $2 \mathrm{~h}$ serum starvation before transfection showed similar transfection efficiency. Nevertheless, 2h FBS-free culture before transfection significantly decreased the cell viability measured by MTT assay $48 \mathrm{~h}$ post-transfection.

Table1: Mean percentage of cell viability and cytotoxicity of HDF cells transfected with different conditions

\begin{tabular}{|lllllllllll|}
\hline & \multicolumn{3}{l}{ DNA Conc. } & \multicolumn{2}{l}{ PEI Conc. } & \multicolumn{2}{c}{ Order } & \multicolumn{2}{c|}{ FBS content } & \multicolumn{2}{c|}{ FBS starvation } \\
\hline Conditions & $1 \mu \mathrm{g}$ & $2 \mu \mathrm{g}$ & Diluted & Conc. & P/D & D/P & FBS+ & FBS- & $1 \mathrm{~h}$ & $2 \mathrm{~h}$ \\
viability & $71^{\star}$ & 62 & $70^{*}$ & 64 & 71 & 72 & $92^{*}$ & 72 & $69 *$ & 57 \\
\hline Cytotoxicity & 29 & 38 & 30 & 36 & 29 & 28 & 8 & 28 & 31 & 43 \\
\hline
\end{tabular}

Conc.: Concentration, P/D: PEI/pDNA, D/P: pDNA/PEI. Asterisk denotes a significant difference $(p<0.05)$

2-2- Determining the best N/P ratio according to the adjusted condition

At the next step, the optimized condition was chosen to measure the transfection efficiency among different N/P ratios of PEI/pGFP complexes (i.e., 3, 4, 6, 8, 10, and 12). GFP-positive cells were determined using flow cytometry and fluorescent microscopy. Our results showed that the transfected cells with N/P ratio of 3 displayed the highest percentage of the cell expressing GFP (an average of $~ 40 \%$ ) (Fig. 2B \& $2 \mathrm{C})$. When we increased the ratio of PEI/pGFP, the amount of GFP-positive HDF significantly decreased in comparison to N/P ratio of $3(p=0.04)$. However, the reduction in transfection efficiency followed by increasing the N/P ratio has not occurred linearly (Fig. 2B \& 2C). Among N/P ratios of 4, 6, 8, 10, and 12, N/P ratio of 4 achieved higher transfection efficiency (29.1\%).

Moreover, the fluorescent microscopy results were consistent with the flow cytometer findings. Fluorescent images represented that the N/P ratio of 3 caused more GFP-expressing cells when compared to N/P ratio of 12 (Fig. 2A).

2-3- Cytotoxicity of PEl complexes 
MTT assay showed that increasing N/P ratio significantly decreased the cell viability index $(p=0.001)$ (Fig. 2D). The cell viability of all HDF groups are represented in table 2 . Results indicated a substantially higher cell survival rate in the HDFs transfected with N/P ratio of 3 than other N/P ratios

Table2: Mean percentage of cell viability and cytotoxicity of HDFs transfected with different N/P ratios

\begin{tabular}{|lllllll|}
\hline N/P ratio & 3 & 4 & 6 & 8 & 10 & 12 \\
\hline viability & 91 & 86 & 79 & 76 & 72 & 69 \\
\hline Cytotoxicity & 9 & 14 & 21 & 24 & 28 & 31 \\
\hline
\end{tabular}

2-4- Generation and characterization of hiPSCs

Complexes with PEI/pDNA ratio of 3 containing $1 \mu \mathrm{g}$ pDNA, diluted PEI $(40 \mu \mathrm{g} / \mathrm{ml})$, and no FBS complexing by adding PEI to PDNA, which assures a balance of considerable transfection efficiency and acceptable toxicity (Fig. 3), was chosen to further transfect HDFs to investigate the potency of this protocol to generate hiPSCs through transfection with oriP/EBNA1 vector. On day 14, post-transfection colonies emerged. Colonies exhibited hESC-like morphology with refractive edges and three-dimensional growth (Fig. 3A). An alkaline phosphatase assay was performed to distinguish the fully reprogrammed hiPSC colonies. AP staining illustrated that 1-3\% of rounded colonies expressed alkaline phosphatase enzyme. The colonies with hESC colonies morphology could be better noticeable from non-iPS colonies upon passaging and were individually and manually picked for expansion and analysis (Fig. 3A). Under sterile conditions and using a $1 \mathrm{ml}$ syringe, hiPSC colonies were broken into pieces on days 30-35 and transferred onto a $1 \%$ Geltrex-coated 12 -well plate containing $0.5 \mathrm{ml}$ PluriSTEM. Plates were incubated at $37^{\circ} \mathrm{C}, 5 \%$ $\mathrm{CO} 2$ incubator. Colonies were attached to the culture plate 48 hours after colony transferring, and from then on, the medium was replaced every day. When the colonies covered $80-90 \%$ of the surface area of the culture plate, they were considered ready for multiple passaging to obtain hiPSC-lines ready for characterization.

Immunofluorescence staining was administrated for the expanded colonies after 8-10 passages. Immunofluorescence microscopic images displayed the expression of pluripotency markers, including KLF4, OCT4, SOX2, and CMYC (Fig. 3B). Furthermore, real-time PCR analyses showed that all chosen clones expressed the endogenous reprogramming and pluripotency factors of nanog, sox 2 and oct4. Relative expression levels (REI) for these pluripotency genes were markedly increased in the iPSCs compared to the parental HDFs (Fig. 3C and Supplementary data S1 and S2 online). The genetic stability of the hiPSCs, were also confirmed by karyotyping (Fig. 3D). The established hiPSC line exhibited a normal karyotype of $46 \mathrm{XY}$ as confirmed by chromosomal G-band analysis at passages 8-10. Moreover, the differentiation potential of generated hiPSCs were examined using embryonic body formation and spontaneous differentiation (Fig. 3E). The expression of three germs layer markers, including $m s \times 1$ (mesoderm), gata4 (Endoderm), and sox 1 (ectoderm) were checked by real-time PCR analysis. All differentiated markers were upregulated in hiPSC-derived differentiated cells in comparison to progenitor hiPSCs (Fig. 3F). 


\section{Discussion}

The iPSC technology opens up an unlimited source of variable human cell types for regenerative medicine. Administrating economical transfection methods such as cationic polymers provide a simplified platform for large-scale production of iPSCs. This method can be used in all iPSC institutes, especially in the labs facing financial issues. In the current investigation, we represented a cost-effective and optimized protocol for transfecting HDFs through a gold standard reagent (i.e PEI) and oriP/EBNA1-based vectors to produce hiPSCs. Previous evidences have confirmed the capability of PEl to transfect several types of cells such as HEK293[2], mesenchymal[39], neural[40], and embryonic[41] stem cells. However, with the aim of generating hiPSCs, our investigation is of the pioneers setting and standardizing several parameters relevant to HDF transfection process using PEI25K.

Our results revealed that using $2 \mu \mathrm{g}$ pDNA implies a lower transfection rate and higher cell death in comparison to $1 \mu \mathrm{g}$ pDNA, which may be related to the aspect ratio of PEI/pDNA. Higher concentrations of pDNA require a higher amount of PEI for modification and permeabilization of endosomal membrane, which causes a higher cytotoxic effect of polyplexes on cell viability[42, 43]. In addition, regardless of PEI dosage, an excess of pDNA itself can also contribute to cellular toxicity[44, 45]. There are pieces of evidence showing that regardless of the transfection method, the concentration of nucleic acid is also critical for enhancing efficiency and minimizing toxicity [44, 46]. For example, Juckem et al. suggested through empirical testing that an optimal starting point is one microgram per well of a 12-well plate resulting in maximum protein expression and minimal toxicity[44]. However, previous studies reported different efficient amounts of pDNA in transfection procedures, partly due to the different sensitivity of diverse cell types to the dosage and concentration of exogenous nucleic acid[44]. In addition, we found that a lower concentration of PEI (dissolved in a low ionic solvent such as distilled water) could prominently increase both transfection efficiency and cell viability compared to concentrated PEI. Besides the lesser toxic effect of PEI at lower concentrations[47], low-concentrated branched PEI have different formation and hydration properties, facilitating transport and diffusion of the polyplexes through cell membrane[48]. Moreover, we hypothesized that diluted PEI might provide more free branches than a concentrated state, advancing DNA binding affinity and improving cellular uptake[49]. In addition, diluted PEI might form a thicker layer around pDNA than concentrated PEl, which enhances the protonation state of PEI/pDNA polyplex structure and provides an electrostatic and physical barrier to DNases with cationic surfaces [24].

Our results also represented that adding PEI to pDNA can increase transfection efficiency relative to the converse order related in part to the electrostatic interactions between the positive charges of cationic polymers and negative charges of pDNA [50]. Therefore, even subtle changes in the sequence of adding reagents may influence the physicochemical properties and reactivity of reagents and subsequently change the transfection efficiency of the polyplexes[51-53].

Furthermore, we found that the presence of serum in polyplexes significantly decreased transfection efficiency due to the interfering role of serum in dissociating and disrupting the structure of PEI/pDNA complexes [18]. However, the MTT test showed that the existence of FBS prominently increased the cell 
viability, which may be attributed to the potential of serum proteins in absorbing free PEIs[18]. Previous studies also confirmed that the presence of FBS in polycations-based transfection reduces both cytotoxicity and transfection efficiency[54]. Therefore, in the current study, using FBS during polyplex formation was not favored by transfection efficiency and is not recommended in HDF cell type.

Apart from the impact of FBS in the structure of polyplexes, it is evident that FBS in cell culture media before transfection can affect the efficiency of PEI/pDNA delivery to the cells. Serum starvation induces cell cycle synchronization (G0/G1 proliferation delay), which eventually increases transfection efficiency $[55,56]$. Moreover, the competitive interaction of serum proteins and polyplexes with cell surface reduce the efficiency of transfection. Herein, we examined the impact of $1 \mathrm{~h}$ vs. $2 \mathrm{~h}$ serum starvation on HDFs' transfection efficiency and cell viability. We discovered that both groups showed the same transfection efficiency. Yet $2 \mathrm{~h}$ serum starvation decreased cell viability in comparison to $1 \mathrm{~h}$ starvation. Nevertheless, the optimized serum starvation period differs in other studies depending on the studied cell types[57].

Given together, our optimized protocol consists of $1 \mu \mathrm{g}$ pDNA, diluted PEI $(40 \mu \mathrm{g} / \mathrm{ml})$, PEI to pDNA addition sequence, serum-free polyplexes, and $1 \mathrm{~h}$ serum starvation of HDFs. Besides, since N/P ratio plays an essential role in influencing the degree of complexation, particle diameter, transfection efficiency, and cytotoxicity of carriers[34], in the next step, we investigated the effect of N/P ratio on transfection efficiency and cell viability using the optimized protocol. We observed that the PEI/pDNA ratio of 3:1 led to the highest transfection efficiency and cell viability in comparison to other higher N/P ratios representing the acceptable strength of low-dose PEl in successfully carrying a large-sized plasmid ( 20kb) into HDFs.

We hypothesized two assumptions regarding this finding. Our first assumption is related to pDNA binding ability of PEI and particle aggregation. The pDNA dosage for all N/P ratios has been fixed $(1 \mu \mathrm{g})$ while the amount of PEl has been raised for increasing N/P ratio. We assumed that due to higher amounts of PEI and more positive charge in respect to the negative charge of pDNA, the pDNA binding ability of PEI has been increased at higher N/P ratios. Thus, despite the potential of higher buffering capacity at higher N/P ratios, extra condensing of pDNA has been occurred, preventing the easy release of pDNA into cytoplasm of cells[58]. Moreover, at higher N/P ratios, polyplexes might have more challenging cellular uptake due to greater sizes, and more particle aggregation $[58,59]$. In line with our finding, Cheraghi et al. also assessed the effect of different PEI/ DNA (firefly luciferase) N/P ratios of $3,6,12,18,24$ on transfection efficiency of MCF7 and BT 474 Cells. They observed a decrease (approximately more than 5 fold) in transfection efficiency from N/P ratio of 12 to 18 [58]. They also interpreted the occurrence of this difference to be as a result of higher particle aggregation, and consequently more challenging cellular uptake at higher N/P ratios. In addition, pDNA size may also have a role in either the mechanism of pDNA release from cationic reagent or intracellular migration of pDNA from the cytoplasm to nucleus[60]. In our study plasmid size was approximately high ( 20kb), adding further challenges regarding particle aggregation at higher N/P ratios[61].

Our second assumption is related to the observed relative higher cytotoxic effect of PEl at higher N/P ratios in the current study. We assumed that even though the transfection might occur for a substantial number 
of cells at N/P ratios greater than 3 (e.g. 4, 6, 8, 10, and 12), but due to higher toxic effects of polyplexes at higher N/P ratios, the number of dead cells had been also relatively increased. Therefore, when we washed the transfected cells for flow cytometry analysis, dead cells (even transfected ones) were removed and washed from the plate and subsequently did not enter the flow cytometry analysis.

The maximum efficacy at a low-dose N/P ratio found in this study differs from previous studies, which used higher polyplex N/P ratios to improve the transfection efficiency even at the cost of cell viability[18, $34,58,62]$. For example, Cheraghi et.al examined the effect of different N/P ratios $(3,6,12,18,24)$ on transfection efficiency and cell viability of MCF7 and BT 474 Cells[58]. They observed significant cytotoxicity of polyplexes in N/P ratios greater than 3 . They reported that according to Dynamic Light Scattering (DLS) results, by increasing N/P ratio, polyplexes showed more positive charges, which in turn produced more transfection efficiency and less cell viability. They postulated that among ratios of $3,6,12$, 18,24 , N/P ratio of 12 could establish an optimized ratio between transfection efficiency and cytotoxicity of PEl/plasmid nanoparticles. In another investigation, Zhang et al. assessed the effect of extracellular nanovesicles (EVs) in PEl-mediated transfection of GFP-encoding plasmid into HEK293T cells, A549 cells, and in the zebrafish embryos[62]. They suggested a very low transfection efficiency for PEI25K and PEI60K at N/P ratios under 32. Due to the negative charge of both EV and DNA, the authors suggested that by increasing N/P ratio higher than 32, zeta potential (surface charge of polyplexes) increases, and subsequently, transfection efficiency will enhance. However, to achieve an acceptable transfection efficiency, they also observed an unavoidable decrease in cell viability by increasing N/P ratio.

We concluded that there could be two possible explanations regarding our finding of acceptable etransfection efficiency in a low N/P ratio. First, our optimization led to significant transfection even at a low N/P ratio of 3 . In line with our results, evidence shows that the complex formation protocol can strongly influence transfection efficiency and cytotoxicity[33]. In other words, reagent/DNA ratio and complex formation conditions is of paramount importance for obtaining maximum efficacy with the least side effects in cell transfection process [63]. Second, in the current study, we used low ionic solvent to dissolve PEI. Evidence have shown that PEI/pDNA complexes in high ionic conditions (e.g. HEPES buffered $150 \mathrm{~mm}$ saline (HBS)) exhibited a strong tendency for aggregation at low N/P ratios, which can be avoided by increasing the N/P ratio to 6 or higher[33]. While polyplexes in low ionic solvents represented an opposite behavior[33]. Therefore, by administrating the optimized conditions and N/P ratio which we presented in the current study, we circumvented issues with either transfection success or high cytotoxicity.

Finally, PEl polyplexes containing oriP/EBNA1 vector were used to generate iPSCs from HDFs through the optimized condition and N/P ratio. In this study, the efficiency of colony formation was about 2 colonies per 150000 input cells. Although efficiency was low, it is still sufficient to establish iPSC lines from HDFs [38]. In the same vein, it has been previously denoted that despite the development of numerous methods of introducing the reprogramming factors into the somatic cells, only a small percentage of cells may completely express the pluripotency factors and end up the road toward pluripotency state[64]. Our colonies exhibited positive AP staining indicating that iPSC colonies had been successfully developed. 
Moreover, immunofluorescence staining and real-time PCR analysis demonstrated the presence of pluripotency-associated proteins including OCT4, CMYC, SOX2, KLF4 proteins and mRNAs including nanog, sox2, oct4. Successively, it was shown that cells could differentiate into different cell types from the three germ layers. The obtained hiPSCs showed a normal karyotype of $46 \mathrm{XY}$ as demonstrated using chromosomal G-band analysis. These results suggest that hiPSC lines were successfully generated using our optimized PEI25K protocol.

It is noteworthy that other groups demonstrated other multiple transfection methods and reagents such as nucleofection[38], Lipotransfection[65], and FuGENE6/HD[25] for transfecting human fibroblasts to generate hiPSCs administrating oriP/EBNA1 or episomal vectors. Yu et al. described the derivation of hiPSCs using oriP/EBNA1 vectors via nucleofection[38]. Similar to our finding, they reported a low reprogramming efficiency (3-6 colonies/ 1000000 input cells). Wang et. al also generated hiPSCs via nucleofection and Binary Colloidal Crystals (BCC) as a feeder free system[66]. Although the authors reported a facilitating role of BCC on reprogramming process and emerging fully reprogrammed hiPSCs colonies, the nucleofection technique requires relatively expensive equipment and reagents[67]. Moreover, Skrzypczyk et al. successfully transferred oriP/EBNA1 vectors into human foreskin fibroblasts through lipotransfection and generated hiPSCs[65]. However, despite the common administration of lipotransfection in the generation of iPSCs, there is evidence showing that lipofection has its own adverse effects on transfected cells[68]. For example, evidence has been demonstrated that lipid-based methods have been linked with causing cell cycle arrest, proinflammatory reactions, and increased apoptosis[68]. In addition, the overall manufacturing cost of cell transfection is also a crucial factor for determining the transfection method[63]. Soe et al. compared three transfection reagents, including lipofectamine 2000, TransIT-PRO, and linear 25KD PEI, regarding cost-effectiveness and efficacy of transient expression of enhanced GFP in Chinese hamster ovary cells[63]. They observed a higher number of mRNA copies per viable cell and higher rProtein titre in PEI than lipofectamine group. They also stated that the cost of lipofectamine reagent would be the highest followed by TransIT-PRO and then PEI. Two grams of PEI supplies enough reagent for 2 Liter solution or 20.000 reactions ( 6 well plate)[41]. For the same price, a very lower number of reactions $(\sim 60)$ can be performed, if expensive reagents such as Lipofectamine are used. In fact, using Lipofectamine imposes much more cost on labs. So, PEl could serve as a cost-effective alternative for large-scale generation of iPSCs which can be a serious limiting factor for labs facing financial problems.

Despite different valuable investigations on PEI properties, to our knowledge, there was no study using PEI25K as a successful carrier for transfecting human fibroblast using oriP/EBNA1 vectors for obtaining hiPSCs. Herein, we introduce a cost-effective and straightforward method to use PEI/pDNA polyplexes in generating iPSCs. In this protocol, we overcome the cytotoxicity of PEl-mediated complexes via optimizing transfection conditions and minimizing PEI/pDNA ratio. Our method can be greatly useful in research projects, especially for labs with financial issues. Availability and Simplicity use of PEI reagents makes it a suitable candidate for large-scale transfection approaches.

\section{Conclusion}


In this study, we represented that PEI25K can transfect HDFs to generate hiPSCs with minimal cytotoxicity. Owing to high molecular weight (>25 KDa), PEl exhibits toxicity issues which are in part due to the absorption of anionic serum proteins onto polyplex surface between cationic polymers and anionic plasma proteins of serum[69]. However, we assumed that a low-dose ratio and an optimized polyplex preparation condition helped minimize polyplexes' toxicity effects on our cell type studied (i.e., HDFs). Our protocol is safer, less costly, and less complicated than conventional viral and lipid-based non-viral methods. Nevertheless, it is worthy to note that some variables other than those aforementioned here may also influence the way polyplexes behave and consequently transfection efficiency and cytotoxicity. We recommend future studies to optimize different parameters relevant to the transfection process with the aim of hiPSCs generation. In sum, using our optimized PEI mediated transfection method is highly recommended, especially in large-scale transfection programs, as an alternative to more expensive commercial reagents for generating hiPSCs. In addition, we could propose that by setting the vital parameters, using PEI on iPSC generation will be a very important primary step toward the extension of the field. At the later phases, we can further improve and optimize PEI efficacy through PEl derivatives for iPSC generation.

\section{Materials And Methods}

This study was approved by Ethics Committee of cellular and molecular research center, Iran University of Medical Sciences (IUMS). All methods were carried out in accordance with Iranian guidelines and regulations regarding the generation of hiPSCs following a protocol approved by Royan Institute.

\section{4-1- Human Dermal Fibroblast culture}

Primary HDFs were purchased from Royan Institute, Stem Cell Biology and Technology Department [35, 36]. The cells were isolated from a skin biopsy of a healthy 37-year-old Iranian male who donated a skin biopsy in reprogramming studies and iPSC lines generation. HDFs were maintained in a medium containing Dulbecco's modified Eagle's medium (DMEM; Gibco), 10\% fetal bovine serum (FBS, Gibco), 100 units/ml penicillin, and $100 \mu \mathrm{g} / \mathrm{ml}$ streptomycin (Pen/Strep) (Gibco). They were at passage four at the time of transfection. Informed consent was obtained from the donor.

\section{4-2- PEl/pGFP (Green Fluorescent Protein) complexes}

One mg branched PEI (molecular weight of 25KDa) was purchased from Polysciences Inc. and dissolved in $1 \mathrm{ml}$ sterile water and filtered with a $0.25 \mu \mathrm{m}$ syringe filter. GFP plasmid (pGFP) (Addgene \#64904) was used to detect the most efficient PEI-mediated gene delivery [37]. Then, immunofluorescence imaging and flow cytometry were performed to detect transfected cells. At the first step, we used N.P ratio of 12 to test different conditions and to find the best-optimized protocol relevant to complex formation. For this purpose, pDNA concentration, PEI concentration, order of combining reagents, FBS content of complexes, and duration of serum starvation prior to transfection were adjusted (See Table 3 for more details on the different conditions of complex formation). Based on the optimized protocol of complex formation, we assessed the impact of different N/P ratios on transfection efficiency and cell toxicity. We prepared 
different N/P ratios of PEI/pGFP complexes, including $3,4,6,8,10$, and 12 . For this purpose, we re-diluted $1,1.44,2.16,2.88,3.6$, and $4.3 \mu \mathrm{l} \mathrm{PEI} 25 \mathrm{~K}(1 \mathrm{mg} / \mathrm{ml})$ in distilled water to the final volume of $25 \mu \mathrm{l} .1 \mu \mathrm{g} / \mathrm{ml}$ of pGFP was also diluted in distilled water to the final volume of $25 \mu$. These polyplex ratios were chosen based on the established protocol in our laboratory. Then, the contents of each PEI tube were added to $25 \mu \mathrm{l}$ of pGFP, and tubes were mixed by gentle pipetting or 10 seconds vortexing. The complexes were incubated at room temperature for 30 minutes. Finally, the synthesized complexes were added to HDFs in $250 \mu$ free FBS medium dropwise. After 4h incubation, we replaced the media with fresh HDFs medium.

Table 3: Different conditions of complex formation to reach the best optimized condition

\begin{tabular}{|c|c|c|c|c|c|c|c|c|c|c|}
\hline \multirow[b]{2}{*}{ Conditions } & \multicolumn{2}{|c|}{ pDNA Conc. } & \multicolumn{2}{|c|}{ PEI Conc. } & \multicolumn{2}{|l|}{ Order } & \multicolumn{2}{|c|}{ FBS content } & \multicolumn{2}{|c|}{$\begin{array}{l}\text { FBS } \\
\text { starvation }\end{array}$} \\
\hline & $1 \mu \mathrm{g}$ & $2 \mu \mathrm{g}$ & $\begin{array}{l}1 \\
\mathrm{mg} / \mathrm{ml}\end{array}$ & $\begin{array}{l}40 \\
\mu \mathrm{g} / \mathrm{ml}^{*}\end{array}$ & $\begin{array}{l}\text { PEI/ } \\
\text { pDNA }\end{array}$ & $\begin{array}{l}\text { pDNA/ } \\
\text { PEI }\end{array}$ & FBS+ & FBS- & $1 \mathrm{~h}$ & $2 \mathrm{~h}$ \\
\hline
\end{tabular}

Note: Conc.: Concentration. PEI/pDNA: PEl added to content of pDNA micro-tube. pDNA/PEl: pDNA added to content of PEI micro-tube. ${ }^{*} \mathrm{PEl}$ concentration of $40 \mu \mathrm{g} / \mathrm{ml}$ was obtained by diluting PEl in distilled water in a ratio of 1:25 (pGFP volume adjusted to the PEI volume in each group to keep the N/P ratio of 12 .

4-3- Transfection Efficiency assay: Immunofluorescence imaging and Flow cytometry

Transfection efficiency of different conditions and N/P ratios were assessed by immunofluorescence imaging under GFP filter and flow cytometry technique $48 \mathrm{~h}$ post-PEI/pGFP transfection. The transfection medium was removed for flow cytometry analysis, and the cells were washed with phosphate-buffered saline (PBS). The cells were harvested by $0.25 \%$ trypsin. The gating was performed based on the untransfected cells considering as the control group. The GFP-positive HDFs were investigated statistically to determine the efficiency of gene transfection. Finally, The data were analyzed with Flow Jo software version 10. All tests were performed in triplicate.

\section{4-4- Cell cytotoxicity evaluation: MTT assay}

The impacts of different conditions and N/P ratios on cellular viability were evaluated by MTT (3-(4,5dimethylthiazol-2-yl)-2,5-diphenyltetrazolium bromide) reduction assay. HDFs were seeded and incubated for $24 \mathrm{~h}$ in a 96 -well plate at a density of $8 * 10^{3}$ per well in DMEM culture media supplemented with $10 \%$ FBS. The next day, each well were treated by PEI-pDNA transfection solutions for $4 \mathrm{~h}$. Thereafter, the solutions replaced with $100 \mu \mathrm{L}$ of DMEM with $10 \%$ FBS. Un-transfected cells were considered as negative controls. All the samples were incubated for $24 \mathrm{~h}$. The next day $10 \mu \mathrm{l}$ of MTT reagent $(5 \mathrm{mg} / \mathrm{ml})$ was added to each well for $4 \mathrm{~h}$. Then, the medium in each well was removed and replaced by $100 \mu \mathrm{l}$ of DMSO. The absorbance at $580 \mathrm{~nm}$ was recorded by ELISA microplate reader (Bio-Rad, Hercules, CA, USA). The cell viability was reported in terms of the control group (considered 100\%) and was calculated based on the following formula: cell viability $(\%)=(O D$ of polymer-treated sample /OD of untreated sample $) * 100$. The data are shown as the mean value \pm Standard Error of the Mean (SEM). Each treatment was run in triplicate. 
The transfection was performed based on the optimized protocol described earlier. Briefly, the $4^{\text {th }}$ passage of HDFs were seeded on Geltrex (Life Technologies) coated 24-well plates the day before transfection. When HDFs reached $70-80 \%$ confluence, they were transfected by oriP/EBNA1-based vector genes presented in Fig. 4 (Plasmid information and sequences are available from Yu et al. supplementary material[38]). Based on the optimized protocol and N/P ratio, we prepared PEI/pDNA complex via diluting 1 $\mu \mathrm{PEI}(1 \mathrm{mg} / \mathrm{ml})$ in distilled water to the volume of $25 \mu \mathrm{l}$ and concentration of $40 \mu \mathrm{g} / \mathrm{ml}$. We also diluted the $1 \mu \mathrm{g}$ pDNA in distilled water to reach the volume of $25 \mu \mathrm{l}$. The contents of the PEl tubes and pDNA tubes were mixed by gentle pipetting. The reagents were incubated at room temperature for 30 minutes. The solution of the PEI/pDNA complexes was added to $250 \mu \mathrm{l}$ starved culture (without FBS and Pen/Strep) and added in a dropwise manner directly to cells grown in four wells of a 24-well plate. After incubating for 4 hours, the medium was replaced with fresh high glucose DMEM with $10 \%$ FBS. Following this, the medium was replaced every other day. On day 4 post-transfection, the morphology of HDFs changed from spindle to rounded shape. At this time, the medium was replaced with human embryonic stem cell culture media (PluriSTEM, Millipore) containing fresh $100 \mathrm{ng} / \mathrm{ml}$ basic-fibroblast growth factor (bFGF, Royan Institute). From then on, the medium was refreshed every day. Human iPS colonies emerged 18-25 days after transfection.

4-6- iPSC characterization

4-6-1 Alkaline phosphatase (AP) staining

Alkaline Phosphatase Staining Kit (Stemgent) was performed according to the manufacturer's protocol to confirm generated iPSCs. In brief, hiPSC colonies were fixed at room temperature for 2 minutes. Then, fixed colonies were treated with AP substrate solution and incubated at room temperature for 15 minutes. Images were captured by Olympus CKX41 microscope with an Olympus DP72 camera. (magnification $\times 100)$.

\section{4-6-2 Immunofluorescence staining}

iPSCs colonies were fixed by $4 \%$ paraformaldehyde for 15 minutes at room temperature and washed 3 times with PBS. Then the cells were treated with $0.2 \%$ Triton X-100 for 15 minutes at room temperature and blocked using $5 \%$ bovine serum albumin (BSA) and $0.1 \%$ Triton $\mathrm{X}-100$ for $1.5 \mathrm{~h}$ at room temperature. Following removal of blocking buffer, colonies were incubated with primary antibodies against KLF4 (ab26648), OCT4 (ab18976), SOX2 (sc-365964), and cMYC (sc764) at $4^{\circ} \mathrm{C}$ overnight. The next day, colonies were washed with PBS three times, and they were incubated with secondary antibodies conjugated with (Alexa Fluor 488) (1:500; Invitrogen; Thermofisher Scientific, Inc.) in the dark for 1 hour at room temperature. After three washing steps, DAPI was applied to stain the cell nuclei. Images were captured within 30 minutes using Olympus BX50 fluorescent microscope.

4-6-3 Cytogenetic analysis (Karyotype) 
The normal chromosomal constitution was verified by conventional karyotyping of generated iPSCs. Human iPSCs were incubated with $10 \mu \mathrm{g} / \mathrm{ml}$ Colcemid (Sigma-Aldrich) for 1 hour after at least 8 passages. After washing with PBS, cells were incubated with $0.075 \mathrm{M} \mathrm{KCl}$ for 20 minutes at $37^{\circ} \mathrm{C}$. Cell fixation was performed by administrating methanol/glacial acetic acid solution (3:1). Conventional cytogenetic analysis was performed on hiPSCs using conventional QFQ-banding at 450 bands resolution according to the International System for Human Cytogenetic Nomenclature. A minimum of 10 metaphase spreads were analyzed for each sample and karyotyped using a chromosome imaging analyzer software (Chromowin software, Tesi Imaging).

4-6-4 Embryonic body (EB) formation

The hiPSCs were harvested using Dispase ( $1 \mathrm{mg} / \mathrm{ml}$, StemCell technologies) when they reached $80-90 \%$ confluence. Then the cells were re-suspended in differentiation medium (high glucose DMEM, $2 \mathrm{Nm}$ Lglutamine, $0.1 \mathrm{Mm}$ nonessential amino acid, $0.1 \mathrm{Mm}$ b-mercaptoethanol, 20\% FBS) and incubated at $37^{\circ} \mathrm{C}$ with $5 \% \mathrm{CO} 2$. The hiPSCs were cultured in suspension, and the medium was refreshed every 2 days. After 1 week, EBs had formed. The following day, EBs were transferred to $1 \%$ Geltrex-coated 6-well plates and cultured in the high glucose DMEM media for 7 days. Finally, the cells were harvested, and gene expression was evaluated by real-time PCR technique. Table 4 represents the primer sequence used for real-time PCR analysis in this study.

\section{4-7- Real-time PCR analysis}

Real-time PCR was performed to evaluate the expression level of pluripotency genes (oct4, sox2, nanog) to confirm generated iPSCs lines. Moreover, gata4, msx1, and sox 1 genes were checked in hiPSC deriveddifferentiated cells to ensure trilineage differentiation (endoderm, mesoderm, and ectoderm, respectively) (Table 4). Total RNA was isolated using TRIzol reagent (Life Technologies). RNA concentrations were examined with NanoDropTM spectrophotometer (Thermo Fisher Scientific), and the 260/280 and 260/230 $\mathrm{nm}$ absorbance ratios were calculated to measure RNA purity. Then $1 \mu \mathrm{g}$ of RNA from each sample was used to synthesize cDNA using cDNA Reverse Transcription Kit (SuperScriptTM III First-Strand Synthesis). All genes were assessed in duplicate by the LightCycler 480 Instrument (Roche) using LightCycler 480 SYBR Green I Master (Roche). For the reaction conditions, initial activation was performed at $95^{\circ} \mathrm{C}$ for 15 min, followed by 45 cycles of thermal denaturation at $95^{\circ} \mathrm{C}$ for $10 \mathrm{~s}$, and annealing and elongation at $60^{\circ} \mathrm{C}$ for $5 \mathrm{~s}$. followed by $72^{\circ} \mathrm{C}$ for $10 \mathrm{~s}$. The cDNA of non-transfected fibroblasts was used as negative controls. Two $\mu$ l of diluted cDNA (1:4) for each sample was added as the real-time PCR reactions template. The realtime PCR was performed with appropriate primers for exogenous pluripotency markers and lineage-specific markers. The sequences of all primers are shown in Table 4. The relative expression level of $\beta$ actin was used for normalization.

Table 4: Real time PCR primer sequences 


\begin{tabular}{|lllll|}
\hline Gene & Forward primer & Reverse primer & $\begin{array}{c}\text { Size } \\
\text { (bp) }\end{array}$ & Accession \# \\
\hline oct4 & GTACTCCTCGGTCCCTTTCC & CAAAAACCCTGGCACAAACT & 168 & NM_001285986.2 \\
\hline nox2 & ACAGCATGATGCAGGACCAG & GACTTGACCACCGAACCCAT & 226 & NM_003106.4 \\
\hline gata4 & GACAATCTGGTTAGGGGAAGC & GAGAGATGCAGTGTGCTCGT & 105 & NM_001308093.3 \\
\hline msx1 & TGCCTCGCTCTACGGTGCCT & GGCTGGAGGAATCGGCTGGC & 154 & NM_002448.3 \\
\hline sox1 & TTTCCCCTCGCTTTCTCA & TGCAGGCTGAATTCGGTT & 104 & NM_005986.3 \\
\hline Bactin & CATGTACGTTGCTATCCAGGC & CTCCTTAATGTCACGCACGAT & 250 & NM_001101.5 \\
\hline
\end{tabular}

\section{4-8- Statistics}

Statistical analyses were performed using Graphpad Prism version 8.0.1. All descriptive results were expressed as mean \pm standard error of at least 2-3 independent experiments. $p$ value of $<0.05$ was considered as statistically significant.

\section{Declarations}

\section{Data availability}

All data generated or analysed during this study are included in this published article and its supplementary information files.

Acknowledgment

We wish to thank greatly Dr. J. Kiani for kindly gifting us oriP/EBNA1 vectors. We kindly thank Dr. M. Koruji and Dr. A.R. Pouya for their invaluable assistance with colony picking and troubleshooting. We also are grateful to Dr. H. Baharvand for helpful discussion and guidance. Finally we thank Iran university of medical sciences and Tehran university of medical sciences for funding this research.

\section{References}

1. Yamanaka, S., Induced pluripotent stem cells: past, present, and future. Cell stem cell, 2012. 10(6): p. 678-684.

2. Choi, H.Y., et al., Efficient mRNA delivery with graphene oxide-polyethylenimine for generation of footprint-free human induced pluripotent stem cells. Journal of Controlled Release, 2016. 235: p. 222235.

3. Pandey, A.P. and K.K. Sawant, Polyethylenimine: A versatile, multifunctional non-viral vector for nucleic acid delivery. Materials Science and Engineering: C, 2016. 68: p. 904-918. 
4. Lehrman, S., Virus treatment questioned after gene therapy death. Nature, 1999. 401: p. 517-518.

5. Zhou, Y.-y. and F. Zeng, Integration-free methods for generating induced pluripotent stem cells. Genomics, proteomics \& bioinformatics, 2013. 11(5): p. 284-287.

6. Rony, I., et al., Inducing pluripotency in vitro: recent advances and highlights in induced pluripotent stem cells generation and pluripotency reprogramming. Cell proliferation, 2015. 48(2): p. 140-156.

7. Park, H.Y., et al., Efficient generation of virus-free iPS cells using liposomal magnetofection. 2012.

8. Liu, W.-H., et al., Human induced pluripotent stem cell and nanotechnology-based therapeutics. Cell transplantation, 2015. 24(11): p. 2185-2195.

9. Cao, X., et al., Non-Viral Co-Delivery of the Four Yamanaka Factors for Generation of Human Induced Pluripotent Stem Cells via Calcium Phosphate Nanocomposite Particles. Advanced Functional Materials, 2013. 23(43): p. 5403-5411.

10. Sohn, Y.-D., et al., Induction of pluripotency in bone marrow mononuclear cells via polyketal nanoparticle-mediated delivery of mature microRNAs. Biomaterials, 2013. 34(17): p. 4235-4241.

11. Khan, M., et al., Delivery of reprogramming factors into fibroblasts for generation of non-genetic induced pluripotent stem cells using a cationic bolaamphiphile as a non-viral vector. Biomaterials, 2013. 34(21): p. 5336-5343.

12. Sunshine, J.C., D.Y. Peng, and J.J. Green, Uptake and transfection with polymeric nanoparticles are dependent on polymer end-group structure, but largely independent of nanoparticle physical and chemical properties. Molecular pharmaceutics, 2012. 9(11): p. 3375-3383.

13. Guo, L., et al., Optimizing conditions for calcium phosphate mediated transient transfection. Saudi journal of biological sciences, 2017. 24(3): p. 622-629.

14. D'Mello, S., et al., Characterization and evaluation of the efficacy of cationic complex mediated plasmid DNA delivery in human embryonic palatal mesenchyme cells. Journal of tissue engineering and regenerative medicine, 2016. 10(11): p. 927-937.

15. Lungwitz, U., et al., Polyethylenimine-based non-viral gene delivery systems. European Journal of Pharmaceutics and Biopharmaceutics, 2005. 60(2): p. 247-266.

16. Meleshko, A., et al., Phase I clinical trial of idiotypic DNA vaccine administered as a complex with polyethylenimine to patients with B-cell lymphoma. Human vaccines \& immunotherapeutics, 2017. 13(6): p. 1398-1403.

17. Lázaro-Martínez, J.M., et al., Solid-state studies of the crystalline/amorphous character in linear poly (ethylenimine hydrochloride)(PEl HCl) polymers and their copper complexes. Macromolecules, 2015. 48(4): p. 1115-1125.

18. Forcato, D., et al., Transfection of bovine fetal fibroblast with polyethylenimine (PEI) nanoparticles: effect of particle size and presence of fetal bovine serum on transgene delivery and cytotoxicity. Cytotechnology, 2017. 69(4): p. 655-665.

19. Benjaminsen, R.V., et al., The possible "proton sponge" effect of polyethylenimine (PEI) does not include change in lysosomal pH. Molecular Therapy, 2013. 21(1): p. 149-157. 
20. Nel, A.E., et al., Understanding biophysicochemical interactions at the nano-bio interface. Nature materials, 2009. 8(7): p. 543-557.

21. Wang, P.-Y., et al., Modulation of PEl-mediated gene transfection through controlling cytoskeleton organization and nuclear morphology via nanogrooved topographies. ACS Biomaterials Science \& Engineering, 2017. 3(12): p. 3283-3291.

22. Seo, E.J., et al., Efficient production of retroviruses using PLGA/bPEI-DNA nanoparticles and application for reprogramming somatic cells. PloS one, 2013. 8(9): p. e76875.

23. Rodier, J.T., et al., Linear Polyethylenimine-DNA Nanoconstruct for Corneal Gene Delivery. Journal of Ocular Pharmacology and Therapeutics, 2019. 35(1): p. 23-31.

24. Godbey, W., K.K. Wu, and A.G. Mikos, Size matters: molecular weight affects the efficiency of poly (ethylenimine) as a gene delivery vehicle. Journal of Biomedical Materials Research: An Official Journal of The Society for Biomaterials, The Japanese Society for Biomaterials, and The Australian Society for Biomaterials, 1999. 45(3): p. 268-275.

25. Drozd, A.M., et al., Generation of human iPSCs from cells of fibroblastic and epithelial origin by means of the oriP/EBNA-1 episomal reprogramming system. Stem cell research \& therapy, 2015. 6(1): p. 117.

26. Kunath, K., et al., Low-molecular-weight polyethylenimine as a non-viral vector for DNA delivery: comparison of physicochemical properties, transfection efficiency and in vivo distribution with highmolecular-weight polyethylenimine. Journal of Controlled Release, 2003. 89(1): p. 113-125.

27. Mintzer, M.A. and E.E. Simanek, Nonviral vectors for gene delivery. Chemical reviews, 2009. 109(2): p. 259-302.

28. Neu, M., D. Fischer, and T. Kissel, Recent advances in rational gene transfer vector design based on poly (ethylene imine) and its derivatives. The Journal of Gene Medicine: A cross-disciplinary journal for research on the science of gene transfer and its clinical applications, 2005. 7(8): p. 992-1009.

29. Jiang, D. and A.K. Salem, Optimized dextran-polyethylenimine conjugates are efficient non-viral vectors with reduced cytotoxicity when used in serum containing environments. International journal of pharmaceutics, 2012. 427(1): p. 71-79.

30. Sawant, R.R., et al., Polyethyleneimine-lipid conjugate-based pH-sensitive micellar carrier for gene delivery. Biomaterials, 2012. 33(15): p. 3942-3951.

31. Derouazi, M., et al., Serum-free large-scale transient transfection of $\mathrm{CHO}$ cells. Biotechnology and bioengineering, 2004. 87(4): p. 537-545.

32. Goula, D., et al., Size, diffusibility and transfection performance of linear PEI/DNA complexes in the mouse central nervous system. Gene therapy, 1998. 5(5): p. 712-717.

33. Ogris, M., et al., The size of DNA/transferrin-PEl complexes is an important factor for gene expression in cultured cells. Gene therapy, 1998. 5(10): p. 1425-1433.

34. Zhao, Q.-Q., et al., N/P ratio significantly influences the transfection efficiency and cytotoxicity of a polyethylenimine/chitosan/DNA complex. Biological and Pharmaceutical Bulletin, 2009. 32(4):

p. 706-710. 
35. Chen, F.G., et al., Clonal analysis of nestin-vimentin + multipotent fibroblasts isolated from human dermis. Journal of cell science, 2007. 120(16): p. 2875-2883.

36. Totonchi, M., et al., Feeder-and serum-free establishment and expansion of human induced pluripotent stem cells. International Journal of Developmental Biology, 2009. 54(5): p. 877-886.

37. Ye, L., et al., Nonviral vector-based gene transfection of primary human skeletal myoblasts. Experimental Biology and Medicine, 2007. 232(11): p. 1477-1487.

38. Yu, J., et al., Human induced pluripotent stem cells free of vector and transgene sequences. Science, 2009. 324(5928): p. 797-801.

39. Tong, $\mathrm{H}$. , et al., Polyethylenimine600- $\beta$-cyclodextrin: a promising nanopolymer for nonviral gene delivery of primary mesenchymal stem cells. International journal of nanomedicine, 2013. 8: p. 1935.

40. Shakhbazau, A., et al., Neurons and stromal stem cells as targets for polycation-mediated transfection. Bulletin of experimental biology and medicine, 2011. 151(1): p. 126-129.

41. Bartman, C.M., et al., A simple and efficient method for transfecting mouse embryonic stem cells using polyethylenimine. Experimental cell research, 2015. 330(1): p. 178-185.

42. Liang, W. and J.K. Lam, Endosomal escape pathways for non-viral nucleic acid delivery systems. Molecular regulation of endocytosis, 2012: p. 429-456.

43. Moghimi, S.M., et al., A two-stage poly (ethylenimine)-mediated cytotoxicity: implications for gene transfer/therapy. Molecular therapy, 2005. 11(6): p. 990-995.

44. Juckem, L., Cellular Toxicity Caused by Transfection: Why is it important. 2012.

45. Chopra, S., et al., Investigation of plasmid DNA delivery and cell viability dynamics for optimal cell electrotransfection in vitro. Applied Sciences, 2020. 10(17): p. 6070.

46. Liew, A., et al., Robust, efficient, and practical electrogene transfer method for human mesenchymal stem cells using square electric pulses. Human gene therapy methods, 2013. 24(5): p. 289-297.

47. Zintchenko, A., et al., Simple modifications of branched PEl lead to highly efficient siRNA carriers with low toxicity. Bioconjugate chemistry, 2008. 19(7): p. 1448-1455.

48. Rudolph, C., et al., In vivo gene delivery to the lung using polyethylenimine and fractured polyamidoamine dendrimers. The journal of gene medicine, 2000. 2(4): p. 269-278.

49. Zhou, D., et al., The transition from linear to highly branched poly ( $\beta$-amino ester) s: Branching matters for gene delivery. Science advances, 2016. 2(6): p. e1600102.

50. Lucotti, A., et al., Molecular interactions of DNA with transfectants: a study based on infrared spectroscopy and quantum chemistry as aids to fluorescence spectroscopy and dynamic light scattering analyses. Rsc Advances, 2014. 4(91): p. 49620-49627.

51. Pezzoli, D., et al., Size matters for in vitro gene delivery: investigating the relationships among complexation protocol, transfection medium, size and sedimentation. Scientific reports, 2017. 7(1): p. 1-11.

52. Pan, S., et al., A Serum-Resistant Low-Generation Polyamidoamine with PEI 423 Outer Layer for Gene Delivery Vector. Macromolecular bioscience, 2013. 13(4): p. 422-436. 
53. Zhang, X., et al., The serum-resistant transfection evaluation and long-term stability of gene delivery dry powder based on mesoporous silica nanoparticles and polyethyleneimine by freezing-drying. AAPS PharmSciTech, 2017. 18(5): p. 1536-1543.

54. Pezzoli, D., et al., Transfection efficiency of PEl polyplexes: a matter of complexation protocol, size and sedimentation.

55. Shin, J.-S., et al., Serum starvation induces $G 1$ arrest through suppression of Skp2-CDK2 and CDK4 in SK-OV-3 cells. International journal of oncology, 2008. 32(2): p. 435-439.

56. Tong, J., et al., Serum starvation and thymidine double blocking achieved efficient cell cycle synchronization and altered the expression of p27, p53, bcl-2 in canine breast cancer cells. Research in veterinary science, 2016.105 : p. 10-14.

57. Oya, N., et al., Effects of serum starvation on radiosensitivity, proliferation and apoptosis in four human tumor cell lines with different p53 status. Strahlentherapie und Onkologie, 2003. 179(2): p. 99106.

58. Cheraghi, R., et al., Optimization of conditions for gene delivery system based on PEI. Nanomedicine Journal, 2017. 4(1): p. 8-16.

59. Sharma, V.K., M. Thomas, and A.M. Klibanov, Mechanistic studies on aggregation of polyethylenimineDNA complexes and its prevention. Biotechnology and bioengineering, 2005. 90(5): p. 614-620.

60. Kreiss, P., et al., Plasmid DNA size does not affect the physicochemical properties of lipoplexes but modulates gene transfer efficiency. Nucleic acids research, 1999. 27(19): p. 3792-3798.

61. Campeau, P., et al., Transfection of large plasmids in primary human myoblasts. Gene Therapy, 2001. 8(18): p. 1387-1394.

62. Zhang, Z., et al., Extracellular Nanovesicle Enhanced Gene Transfection Using Polyethyleneimine in HEK293T Cells and Zebrafish Embryos. Frontiers in bioengineering and biotechnology, 2020. 8: p. 448.

63. Sou, S.N., K.M. Polizzi, and C. Kontoravdi, Evaluation of transfection methods for transient gene expression in Chinese hamster ovary cells. Advances in Bioscience and Biotechnology, 2013. 2013.

64. Plath, K. and W.E. Lowry, Progress in understanding reprogramming to the induced pluripotent state. Nature reviews genetics, 2011. 12(4): p. 253-265.

65. Skrzypczyk, A., S. Giri, and A. Bader, Generation of induced pluripotent stem cell line from foreskin fibroblasts. Stem cell research, 2016. 17(3): p. 572-575.

66. Wang, P.-Y., et al., Binary colloidal crystals (BCCs) as a feeder-free system to generate human induced pluripotent stem cells (hiPSCs). Scientific reports, 2016. 6(1): p. 1-11.

67. Zeitelhofer, M., et al., High-efficiency transfection of mammalian neurons via nucleofection. Nature protocols, 2007. 2(7): p. 1692-1704.

68. Sharma, T., et al., Transfection methods affect cellular function and gene expression. Animal Science Papers and Reports, 2018. 36(4): p. 431-451.

69. Wadhwa, A., et al., Opportunities and challenges in the delivery of mRNA-based vaccines. Pharmaceutics, 2020. 12(2): p. 102. 


\section{Figures}
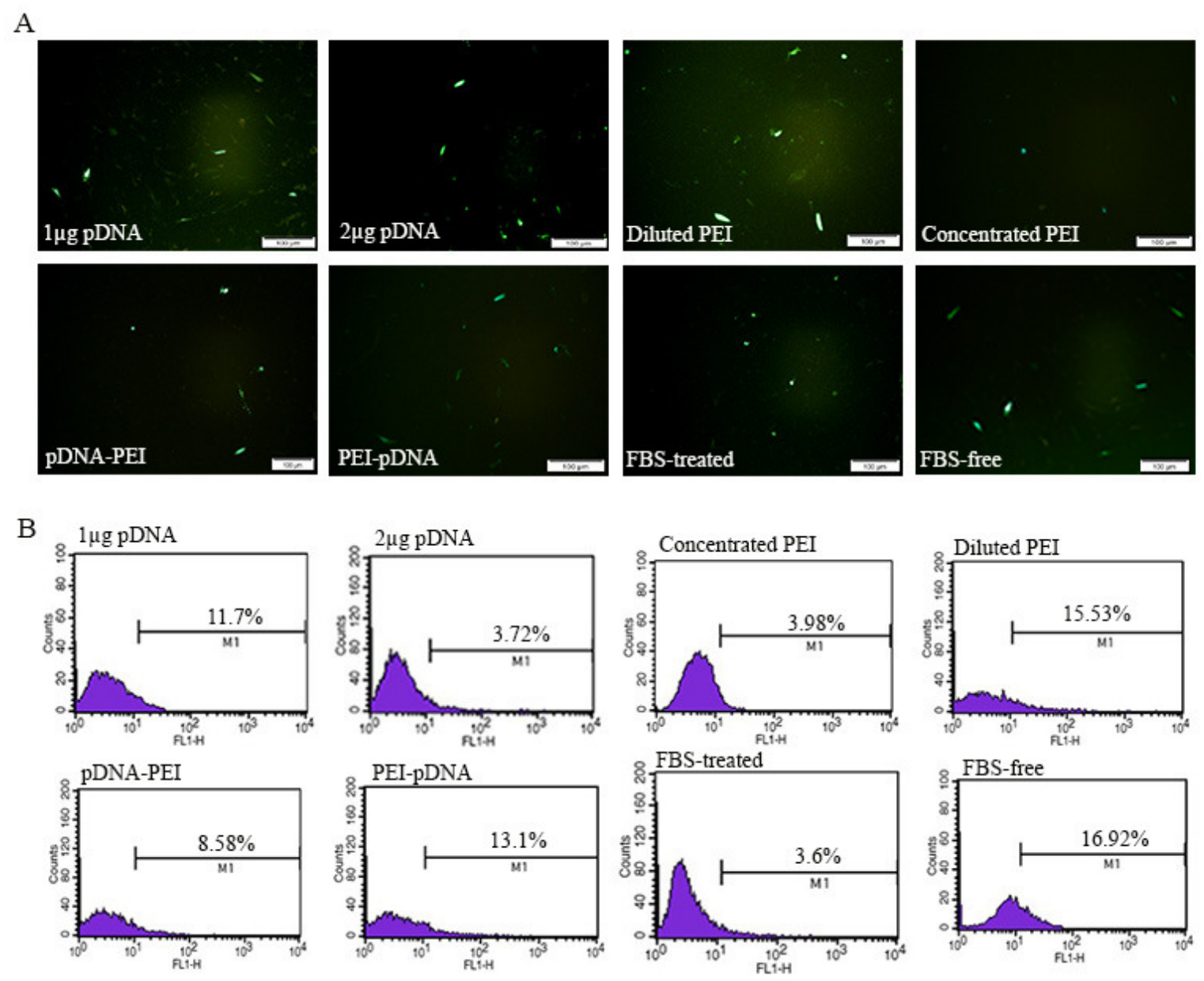

C

D
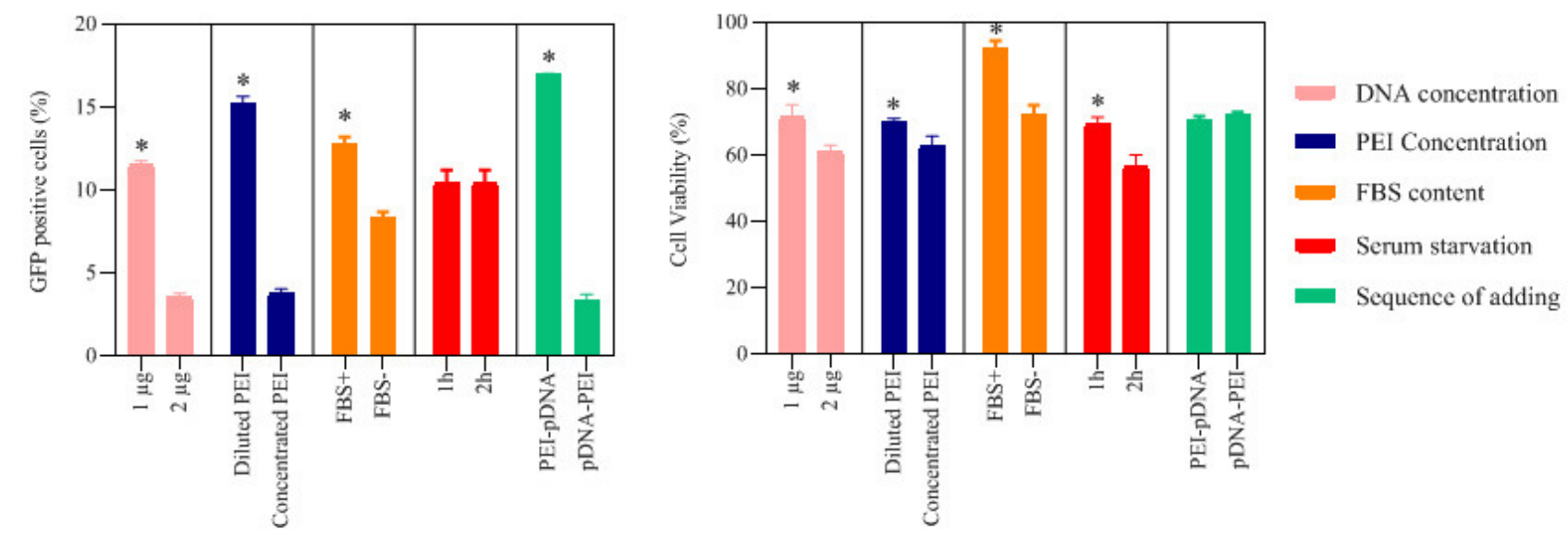

\section{Figure 1}

Initial optimization of PEl transfection conditions. HDF cells were assessed by fluorescence microscopy, flow cytometry, and MTT assay $48 \mathrm{~h}$ after transfection. (A) HDF cells are visualized by fluorescent microscopy. Fluorescent images are shown at 10x magnification under GFP filter. The image illustrates the 
comparative transfection of HDF cells with $1 \mu \mathrm{g}$ vs. $2 \mu \mathrm{g}$ pGFP (upper left), concentrated vs. diluted PEI (upper right), the addition of PEl to pGFP vs. addition of pGFP to PEI for forming complexes (lower left), and PEI/pGFP complexes without FBS vs. with $10 \%$ FBS (lower right). Transfected HDF cells with $1 \mu \mathrm{g}$ pGFP, diluted PEI $(40 \mu \mathrm{g} / \mathrm{ml})$, PEl-added-to-pGFP complexes, and PEI/pGFP complexes without FBS are more in number than $2 \mu \mathrm{g}$ pGFP, concentrated PEI $(1 \mathrm{mg} / \mathrm{ml})$, pGFP-added-to-PEI complexes, and PEI/pGFP complexes with $10 \%$ FBS respectively. (B) Quantification of transfected HDF cells with different conditions through flow cytometry analysis. All analyses were performed on 3 independent HDF transfections. The $x-$ axis denotes fluorescence, and the $y$-axis shows the number of cells detected at a defined fluorescence by the flow cytometer. Untransfected HDFs were used as a reference, and the gating was set such $1.2 \%$ of cells were untransfected. $1 \mu \mathrm{g}$ pDNA (11.7\%), diluted PEI (15.53\%), PEl-added-to-pGFP complexes (13.1\%), and PEI/pGFP complexes without FBS (16.92\%) resulted in more percentage of cells positive for pGFP in comparison to $2 \mu \mathrm{g}$ pDNA (3.72\%), concentrated PEI (3.98\%), pGFP-added-to-PEI complexes (8.58\%), and PEI/pGFP complexes with 10\% FBS (3.62\%) respectively. (C) Error bars represent the standard error of the mean (SEM). This graph displays the percentage of GFP+ cells. The $x$-axis represents the different conditions relevant to complex formation, and the $y$-axis represents the percentage of transfection as determined by flow cytometry (asterisk denotes differences at p $\backslash 0.05$ ). (D) Effect of pDNA concentration, PEI concentration, the sequence of adding reagents, FBS content of polyplexes, and serum starvation before transfection on cell viability measured by MTT assay. The data are expressed as a percentage of the absorbance of untreated cells and presented as the mean of three independent replicates \pm SEM. Asterisk denotes a significant difference $(p<0.05)$. 
A
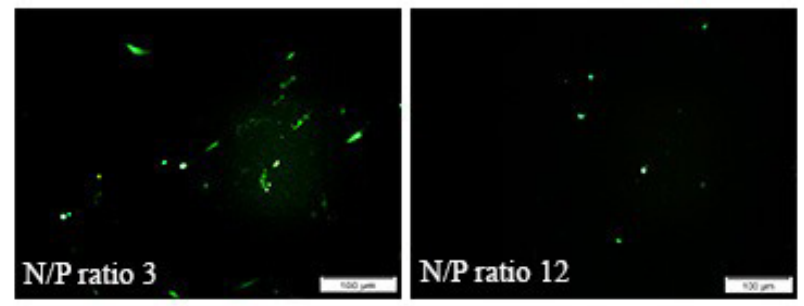

B
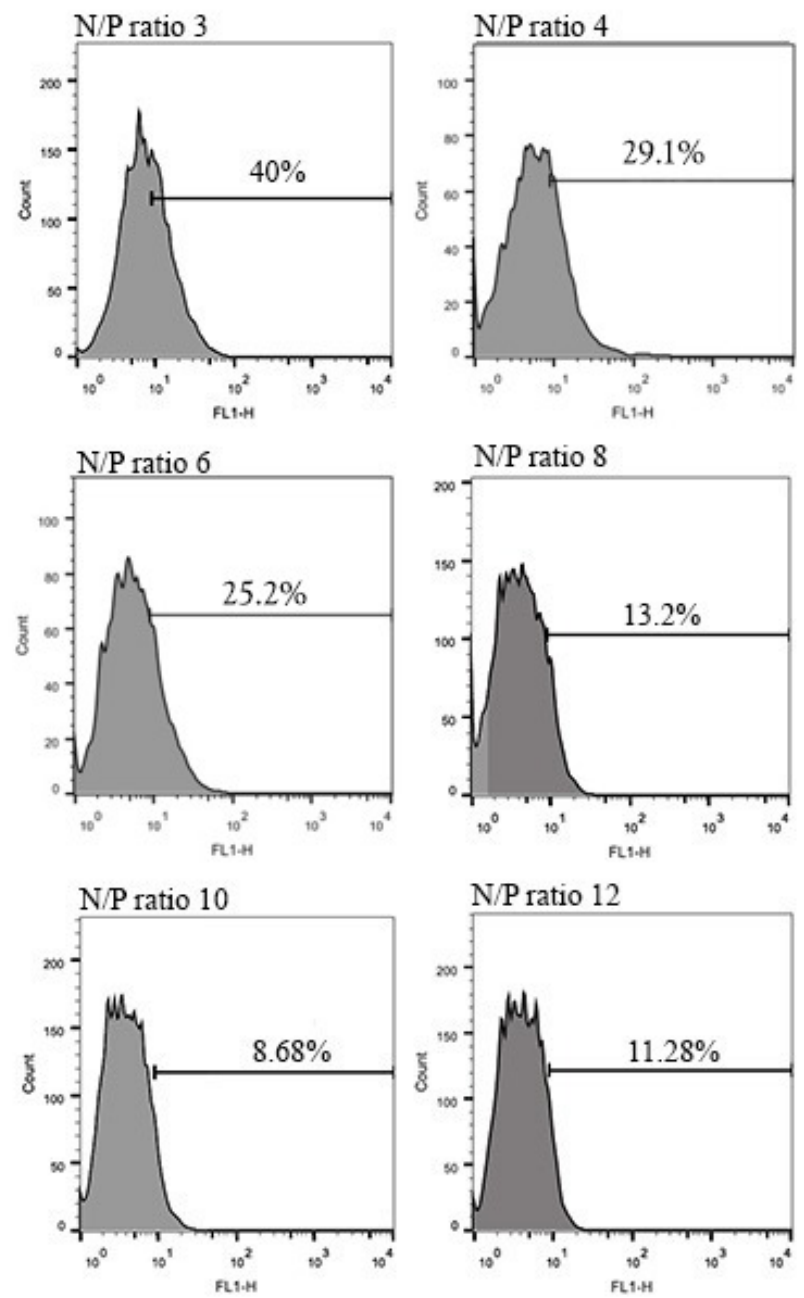

$\mathrm{C}$

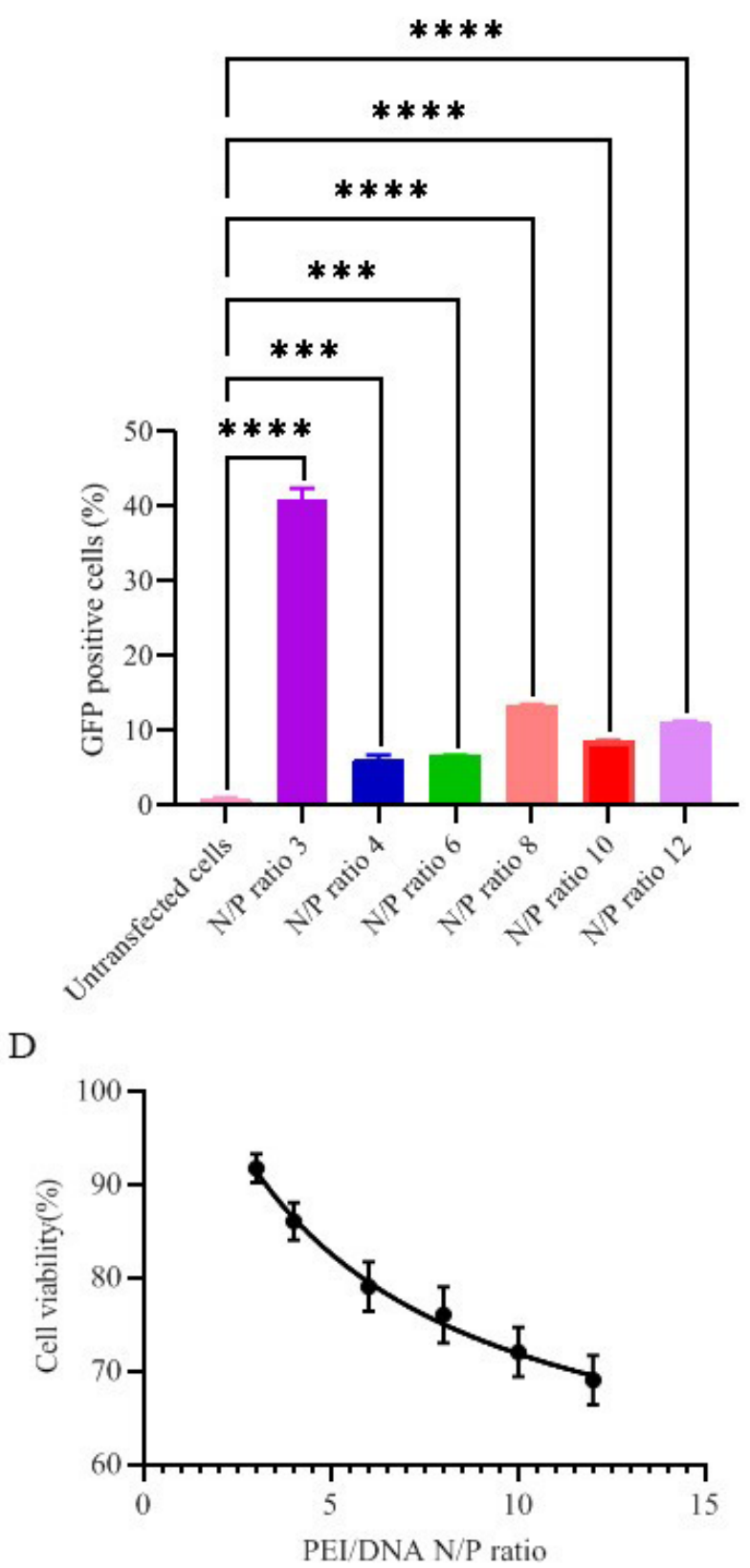

$\mathrm{PEI} / \mathrm{DNA} \mathrm{N} / \mathrm{P}$ ratio

\section{Figure 2}

(A) Optimized PEl protocol using N/P ratio of 3 resulted in more GFP positive cells than N/P ratio of 12 observed under fluorescent microscopy (10x magnification). (B) Quantification of transfected cells; this data represents flow cytometry analysis of HDF transfected with different N/P ratios of $3,4,6,8,10$, and 12 , all in the same optimized conditions. All analyses were performed on 3 independent HDF transfections. The $x$-axis denotes fluorescence, and the $y$-axis shows the number of cells detected at a defined fluorescence by the flow cytometer. Untransfected HDFs were used as a reference, and the gating was set such $0.9 \%$ of cells were untransfected. PEI/pGFP ratios of $3,4,6,8,10$, and 12 resulted in $40.1 \%, 29.1 \%$, $25.2 \%, 13,2 \%, 8.68 \%$, and $11.28 \%$ GFP-positive cells respectively (C) Error bars represent the standard error 
of the mean (SEM). This graph shows the percentage of GFP+ cells determined in (3B). Asterisks denote significance level at $p<0.05$. The $x$-axis represents the N/P ratios compared in this manuscript, and the $y$ axis represents the percentage of transfection as determined by flow cytometry. (D) Cytotoxicity of different N/P ratios. The effect of different complexes with the same optimized condition but different N/P ratios of $3,4,6,8,10$ and 12 on cell viability were measured by MTT metabolism. Results are expressed as a percentage of the absorbance of untreated cells and presented as the mean of three independent replicates \pm SEM. The mean percentage of cell viability at N/P ratios of $3,4,6,8,10$, and 12 were $91 \%, 86 \% 79 \%, 76 \%$, $72 \%$ and $69 \%$ respectively. 
A

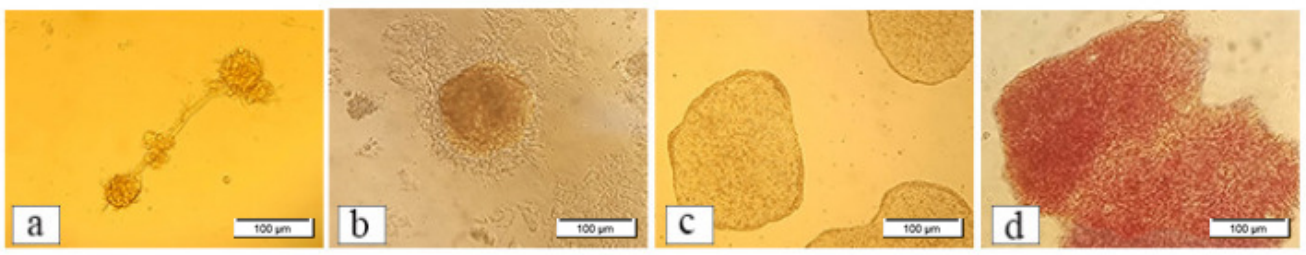

B

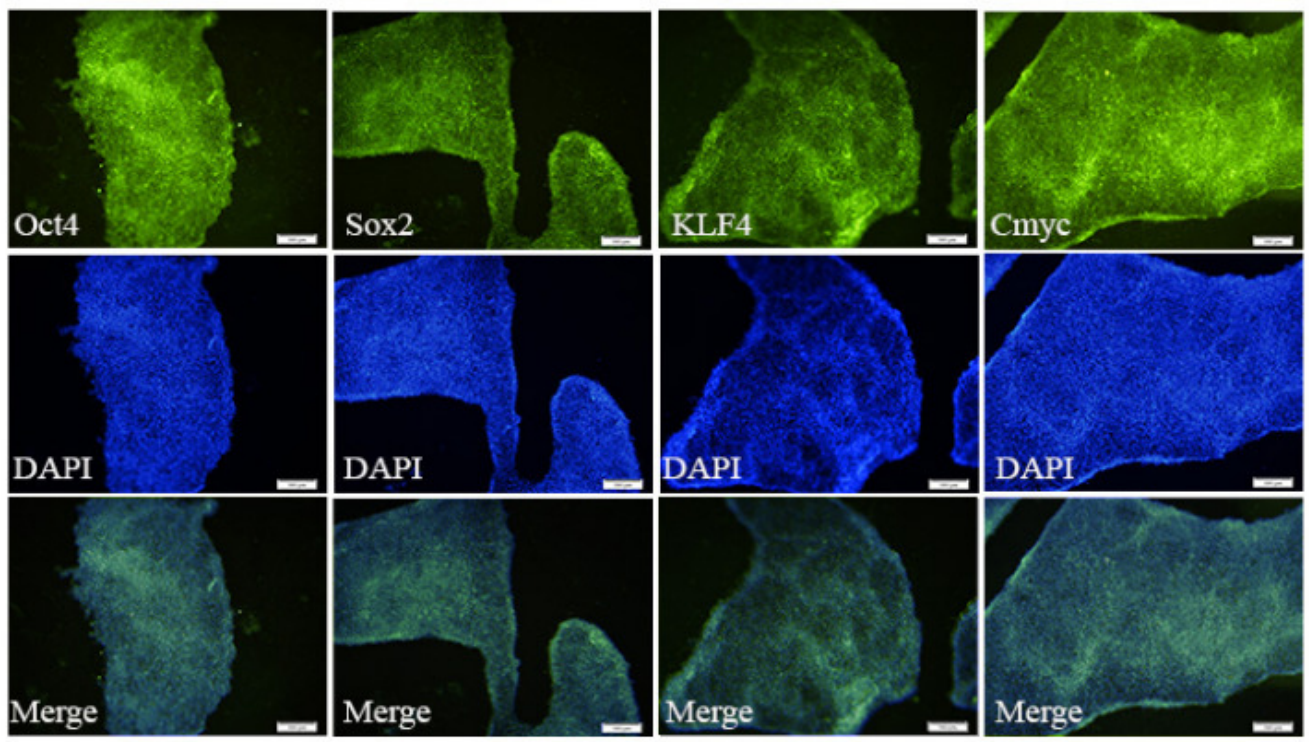

$\mathrm{C}$

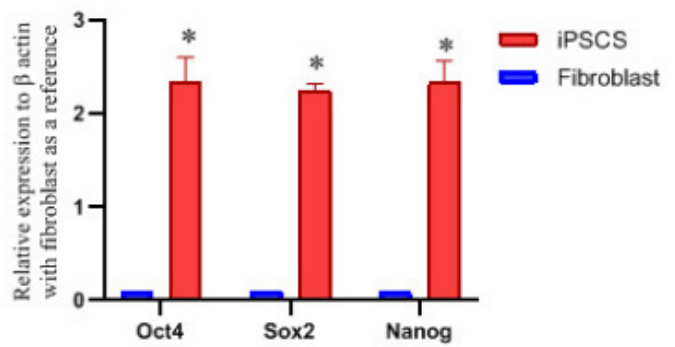

$\mathrm{D}$

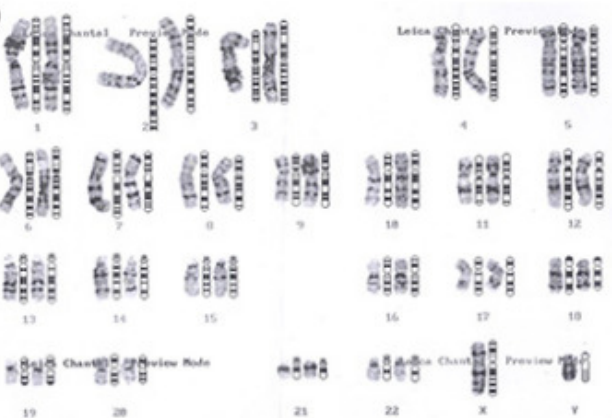

E

$\mathrm{F}$

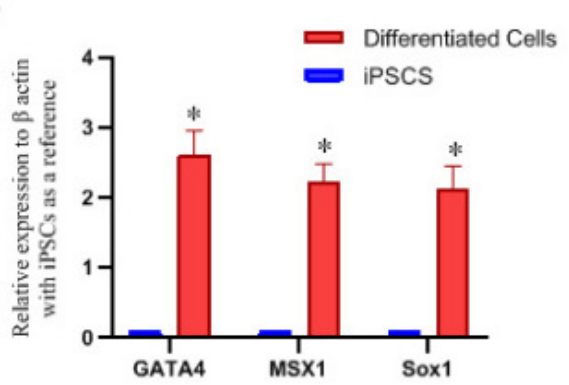

Figure 3

(A) Generation of hiPSCs; Scale bars $100 \mu \mathrm{m}$ (a) Potential small iPSC colonies with morphology similar to ESC colonies at day 14 (b) Potential colonies being increased in size at day 20. These colonies were harvested and passaged at day 25-30 post-transfection to a fresh Geltrex-coated 24-well plate at the ratio of 1:2 with Dispase treatment (c) hiPSCs upon passaging at day 30 post-transfection (d) Positive APstaining of obtained hiPSC line. (B) Immunofluorescence staining was performed at passages 8-10 to 
detect pluripotent markers, including KLF4, OCT4, SOX2, and CMYC. The nuclei of all cells were stained

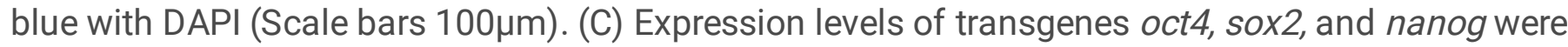
assessed at passages 8-10 by real-time PCR and plotted relative to $\beta$ actin. HDFs were used as negative controls. The error bar indicated standard deviation (SD). (D) Karyotype analysis of HDF-induced pluripotent stem cells. Karyotype analysis revealed a normal karyotype of 46 chromosomes (E) hiPSCs were grown in suspension, and EBs were detected after 8 days. EBs were cultured in Geltrex-coated 6-well plates for an additional 7 days, and cells were checked for their adherent status (Scale bar $200 \mu \mathrm{m}$ ) (F) realtime PCR analysis was performed for differentiation markers of the three germ layers in EBs. ßactin was utilized as an internal control.

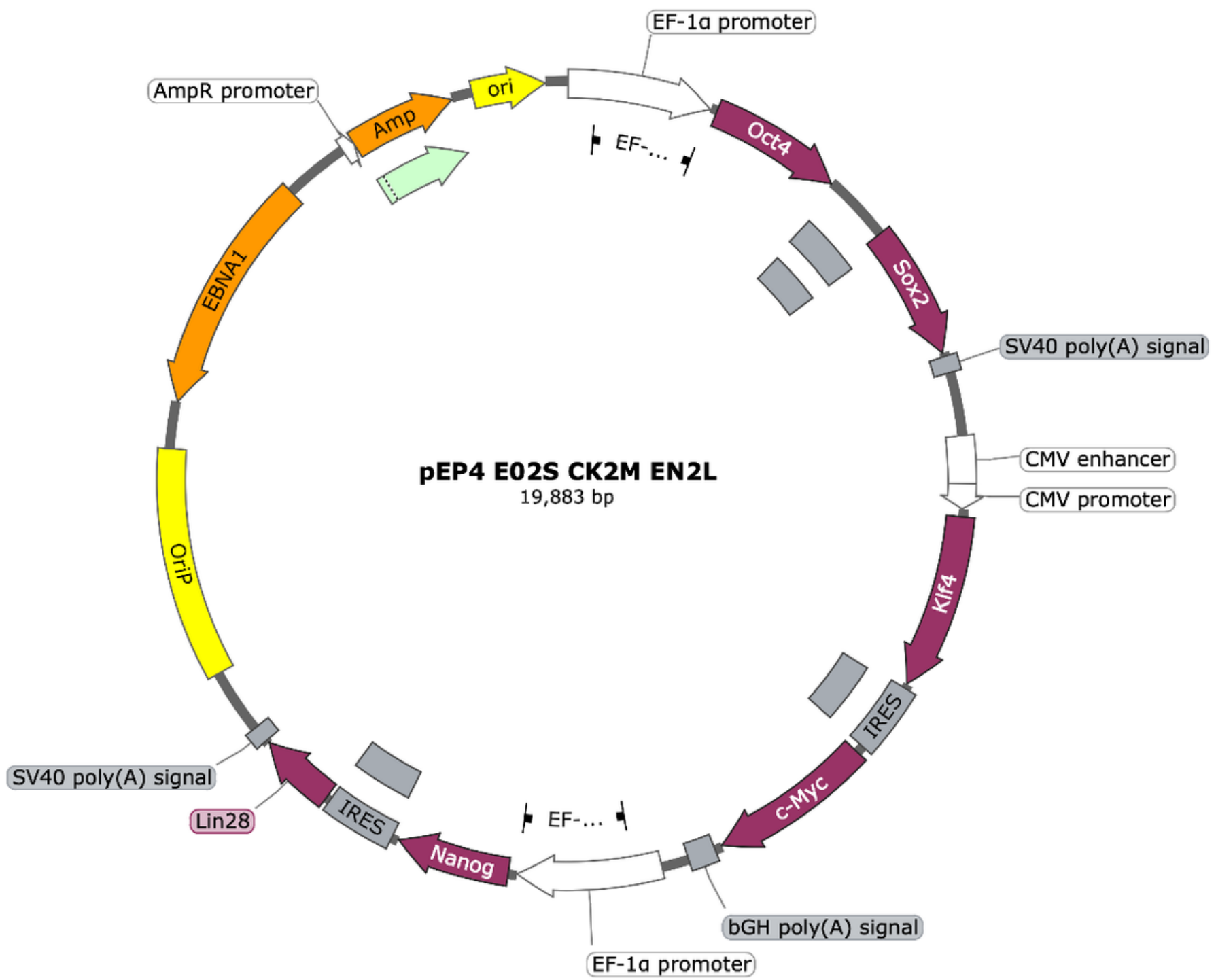

\section{Figure 4}

oriP/EBNA1-based vector genes including OCT4 (NM_00701), SOX2 (NM_003106), NANOG (NM_024865), LIN28 (NM_024674), c-Myc (NM_002467), and KLF4 (NM_004235) 


\section{Supplementary Files}

This is a list of supplementary files associated with this preprint. Click to download.

- RTPCRrawdataS1.rar 